\title{
Some New Inequalities Using Nonintegral Notion of Variables
}

\author{
Abha Singh, ${ }^{1}$ Abdul Hamid Ganie, ${ }^{1}$ and Mashael M. Albaidani $\mathbb{D}^{2}$ \\ ${ }^{1}$ Department of Basic Sciences, College of Science and Theoretical Studies, Saudi Electronic University, Riyadh 11673, Saudi Arabia \\ ${ }^{2}$ Department of Mathematics, College of Science and Humanities Studies, Prince Sattam Bin Abdulaziz University, \\ Al Kharj 11942, Saudi Arabia
}

Correspondence should be addressed to Mashael M. Albaidani; m.albaidani@psau.edu.sa

Received 26 September 2021; Revised 3 November 2021; Accepted 23 November 2021; Published 9 December 2021

Academic Editor: Antonio Scarfone

Copyright (C) 2021 Abha Singh et al. This is an open access article distributed under the Creative Commons Attribution License, which permits unrestricted use, distribution, and reproduction in any medium, provided the original work is properly cited.

The object of this paper is to present an extension of the classical Hadamard fractional integral. We will establish some new results of generalized fractional inequalities.

\section{Introduction}

It is important to note that the integral inequalities play a basic role in statistics, mathematics, sciences, and technology (SMST). As in [1-8], it has proven to be of great importance from the past few decades. The formation of fractional calculus has straight impact on the theory utilizing the solution of various spaces in SMST and to prove its efficacy, various statements and applications of fractional derivatives have been constructed. Authors Riemann-Liouville and Grunwald-Letnikov are well known in this filed. Caputo reformulated the classical statement of the Riemann-Liouville fractional derivative for finding solutions of fractional differential equations using initial conditions. The notion of fractional calculus given by Leibniz was studied by Grunwald-Letnikov in a different structure [9-12].

Recently, in [13-17] and [18], the development between probability theory and fractional calculus was given, and the results of the classical approach were extended. Also, analysis and observations on this direction and several purposes have been found in the concrete problems which include applied mathematics and fluid mechanics as in $[2,18-26]$, and many others.

As in [27-32], for a function $\mathfrak{g}(v) \in L^{1}([\alpha, \beta])$, the Hadamard fractional integral of order $\kappa \geq 0$ is given as follows:

$$
{ }_{H} \mathcal{J}_{\alpha}^{\kappa}[\mathfrak{g}(v)]=\frac{1}{\Gamma(\kappa)} \int_{\alpha}^{v} \ln \left(\frac{v}{u}\right)^{\kappa-1} \mathfrak{g}(u) \frac{d u}{u}, \quad 0<\alpha<v \leq \beta,
$$

which differs from Riemann-Liouville and Caputo's definition in the sense that the kernel of integral (1) contains a logarithmic function of an arbitrary exponent.

We need the following definition while determining some application, and it is called the Beta function.

As in [22], the Beta function, symbolized by $\beta(l, m)$, is given as

$$
\beta(l, m)=\int_{0}^{1} \tau^{l-m}(1-\tau)^{m-1} d \tau=\frac{\Gamma(l) \Gamma(m)}{\Gamma(l+m)} .
$$

The basic notion of generalization of special functions using a kind of new parameter fascinated many researchers and mathematicians. More details about fractional integrals can be found in [33-36] and others as cited in the text. Accordingly, our main scenario in this paper is to extend the idea of a new fractional integration with parameter $\kappa \geq 0$ that generalizes Hadamard fractional integrals. 


\section{Main Results}

In this section, we shall be dealing with the new generalized type of results to random variables of a continuous type of fractional integral order $\kappa \geq 0$.

Definition 1. For a function $\mathfrak{g}(v) \in L^{1}([\alpha, \beta])$, the generalized fractional integral of the Hadamard type with order $\kappa \in \mathbb{R}^{+}$is given by

$$
{ }_{H^{\prime}}^{\mathcal{J}_{\alpha, v}^{-\kappa, \lambda}}[\mathfrak{g}(v)]=\frac{1}{\lambda \Gamma(\kappa)} \int_{\alpha}^{v} \ln \left(\frac{v}{u}\right)^{\kappa / \lambda-1} \mathfrak{g}(u) \frac{d u}{u}, \quad 0<\alpha<v \leq \beta,
$$

where $\Gamma(\kappa)=\int_{0}^{\infty} e^{-u} u^{\kappa-1} d u$ represents the Gamma function as can be seen in $[37,38]$ and many more.

Definition 2. For a r.v. $\mathscr{Z}$ having positive p.d.f. $\mathfrak{g}:[\alpha, \beta]$ $\longrightarrow \mathbb{R}^{+}(\alpha>0)$, we define the fractional expectation function of order $\kappa \geq 0$ as

$$
E_{\mathscr{L}, \kappa, \lambda}(v)={ }_{H} \mathscr{I}_{\alpha, v}^{-\kappa, \lambda}[v \mathfrak{g}(v)]=\frac{1}{\lambda \Gamma(\kappa)} \int_{\alpha}^{v}\left(\ln \frac{v}{u}\right)^{\kappa / \lambda-1} u \mathfrak{g}(u) \frac{d u}{u}
$$

where $\alpha<v<\beta$.

Definition 3. For a r.v. $\mathscr{Z}$ having a positive p.d.f. $\mathfrak{g}:[\alpha, \beta]$ $\longrightarrow \mathbb{R}^{+}(\alpha>0)$, the fractional expectation function of $\mathscr{Z}-E(\mathscr{Z})$ of order $\kappa \geq 0$ is given as

$$
E_{\mathscr{Z}-E(\mathscr{E}), \kappa, \lambda}(v)=\frac{1}{\lambda \Gamma(\kappa)} \int_{\alpha}^{v}\left(\ln \frac{v}{u}\right)^{\kappa / \lambda-1}(u-E(\mathscr{Z})) \mathfrak{g}(u) \frac{d u}{u},
$$

where $\alpha<v<\beta$.

Definition 4 . For a r.v. $\mathscr{Z}$ having a positive p.d.f. $\mathfrak{g}:[\alpha, \beta]$ $\longrightarrow \mathbb{R}^{+}(\alpha>0)$, the fractional expectation function of order $\kappa \geq 0$ is given as

$$
\begin{aligned}
E_{\mathscr{Z}, \kappa, \lambda}(v) & ={ }_{H} \mathscr{J}_{1, v}^{-\kappa, \lambda}[v \mathfrak{g}(v)] \\
& =\frac{1}{\lambda \Gamma(\kappa)} \int_{\alpha}^{\beta}\left(\ln \frac{v}{u}\right)^{\kappa / \lambda-1}(u-E(\mathscr{Z})) \mathfrak{g}(u) \frac{d u}{u} .
\end{aligned}
$$

Definition 5. If $E(\mathscr{Z})$ symbolizes the expected value of the r.v. $\mathscr{Z}$ having a positive p.d.f. $\mathfrak{g}:[\alpha, \beta] \longrightarrow \mathbb{R}^{+}$with $\alpha>0$, then the fractional variance function having order $\kappa$ of $\mathscr{Z}$ is given by

$$
\begin{aligned}
\sigma_{\mathscr{Z}, \kappa, \lambda}^{2} & ={ }_{H} \mathcal{I}_{1, v}^{-\kappa, \lambda}\left[(v-E(\mathscr{Z})]^{2} \mathfrak{g}(v)\right] \\
& =\frac{1}{\lambda \Gamma(\kappa)} \int_{\alpha}^{v}\left(\ln \frac{v}{u}\right)^{\kappa / \lambda-1}(u-E(\mathscr{Z}))^{2} \mathfrak{g}(u) \frac{d u}{u},
\end{aligned}
$$

where $\alpha<v<\beta$.
Definition 6. If $E(\mathscr{Z})$ symbolizes the expected value of the r.v. $\mathscr{Z}$ having a positive p.d.f. $\mathfrak{g}:[\alpha, \beta] \longrightarrow \mathbb{R}^{+}$with $\alpha>0$, then the fractional variance function having order $\kappa$ of $\mathscr{Z}$ is given by

$$
\sigma_{\mathscr{Z}, \kappa, \lambda}^{2}=\frac{1}{\lambda \Gamma(\kappa)} \int_{\alpha}^{\beta}\left(\ln \frac{v}{u}\right)^{\kappa / \lambda-1}(u-E(\mathscr{Z}))^{2} \mathfrak{g}(u) \frac{d u}{u} .
$$

Now by choosing different values of $\kappa$ and $\lambda$, we have the following remarks.

Remark 7. (R1) Choosing $\lambda=1$ and $\kappa=1$, the classical expectation of r.v. $\mathscr{Z}$ will be deduced.

(R2) Choosing $\lambda=1$ and $\kappa=1$, the classical variance of r.v. $\mathscr{Z}$ will be deduced.

(R3) Choosing $\lambda=1$, we reach to the definition of [31].

Theorem 8. Let the continuous r.v. be $\mathscr{Z}$ with p.d.f. $g$ : $[\alpha, \beta]$ $\longrightarrow \mathbb{R}^{+}$. Then,

$$
\sigma_{\mathscr{Z}, \kappa, \lambda}^{2}=E_{\mathscr{E}^{2}, \kappa, \lambda}-2 E(\mathscr{Z}) E_{\mathscr{Z}, \kappa, \lambda}+E(\mathscr{Z})_{H}^{2} \mathscr{J}_{1, v}^{-\kappa, \lambda}[\mathfrak{g}(\beta)] .
$$

for all $\kappa \geq 0$.

Proof. By definition, we have

$$
\begin{aligned}
\sigma_{\mathscr{E}, \kappa, \lambda}^{2}= & \frac{1}{\lambda \Gamma(\kappa)} \int_{\alpha}^{\beta}\left(\ln \frac{v}{u}\right)^{\kappa / \lambda-1}(u-E(\mathscr{Z}))^{2} \mathfrak{g}(u) \frac{d u}{u} \\
= & \frac{1}{\lambda \Gamma(\kappa)} \int_{\alpha}^{\beta}\left(\ln \frac{v}{u}\right)^{\kappa / \lambda-1}\left(u^{2}-2 u E(\mathscr{Z})+E(\mathscr{Z})^{2}\right) \mathfrak{g}(u) \frac{d u}{u} \\
= & \frac{1}{\lambda \Gamma(\kappa)} \int_{\alpha}^{\beta}\left(\ln \frac{v}{u}\right)^{\kappa / \lambda-1} u^{2} \mathfrak{g}(u) \frac{d u}{u} \\
& -2 E(\mathscr{Z}) \frac{1}{\lambda \Gamma(\kappa)} \int_{\alpha}^{\beta}\left(\ln \frac{v}{u}\right)^{\kappa / \lambda-1} u \mathfrak{g}(u) \frac{d u}{u} \\
& +E(\mathscr{Z})^{2} \frac{1}{\lambda \Gamma(\kappa)} \int_{\alpha}^{\beta}\left(\ln \frac{v}{u}\right)^{\kappa / \lambda-1} \mathfrak{g}(u) \frac{d u}{u} \\
= & E_{\mathscr{Z}^{2}, \kappa, \lambda}-2 E(\mathscr{Z}) E_{\mathscr{X}, \kappa, \lambda}+E(\mathscr{Z})_{H}^{2} \mathscr{J}_{1, v}^{-\kappa, \lambda}[\mathfrak{g}(\beta)] .
\end{aligned}
$$

Theorem 9. Define a r.v. $\mathscr{Z}$ having a p.d.f. $g:[\alpha, \beta] \longrightarrow \mathbb{R}^{+}$. Then, we have the following inequalities:

$$
\text { (i) } \begin{aligned}
{ }_{H} \mathscr{J}_{1, v}^{-\kappa, \lambda}[\mathfrak{g}(v)] \sigma_{\mathscr{Z}, \kappa, \lambda}^{2}-\left[E_{\mathscr{E}-E(\mathscr{E}), \kappa}(v)\right]^{2} \leq\|\mathfrak{g}\|_{\infty}^{2}[2 \\
\left.(\ln (v / u))^{\kappa / \lambda} / \Gamma(\kappa+1)_{H} \mathscr{I}_{1, v}^{-\kappa, \lambda}\left[v^{2}\right]-2\left({ }_{H} \mathscr{I}_{1, \nu}^{-\kappa, \lambda}[v]\right)^{2}\right],
\end{aligned}
$$

holds if $g \in L_{\infty}[\alpha, \beta]$ and for all $\alpha<\nu \leq \beta, \kappa \geq 0$, and $\lambda \geq 0$.

$$
\begin{aligned}
& \text { (ii) }{ }_{H} \mathscr{J}_{1, v}^{-\kappa, \lambda}[\mathfrak{g}(v)] \sigma_{\mathscr{E}, \kappa, \lambda}^{2}-\left[E_{\mathscr{Z}-E(\mathscr{I}), \kappa}(v)\right]^{2} \leq 1 / 2(v-\alpha)^{2}{ }_{H} \\
& \mathscr{I}_{1, v}^{-\kappa, \lambda}[\mathfrak{g}(v)]
\end{aligned}
$$

holds. 
Proof. For the proof of the result, we begin by choosing the function $\mathfrak{H}$ for $x, y \in(\alpha, v), \alpha<v \leq \beta$ as follows:

$$
\begin{aligned}
\mathfrak{H}(v, y)= & \left(\mathfrak{H}_{1}(x)-\mathfrak{H}_{1}(y)\right)\left(\mathfrak{S}_{2}(x)-\mathfrak{S}_{2}(y)\right) \\
= & \mathfrak{J}_{1}(x) \mathfrak{S}_{2}(x)-\mathfrak{S}_{1}(x) \mathfrak{H}_{2}(y)-\mathfrak{H}_{1}(y) \mathfrak{H}_{2}(v) \\
& +\mathfrak{H}_{1}(y) \mathfrak{H}_{2}(y),
\end{aligned}
$$

where $\kappa \geq 0$.

Now on both sides of (11), we multiply by $\left((\ln (v / x))^{\kappa / \lambda-1}\right.$ $\mid x \lambda \Gamma(\kappa)) \mathfrak{p}(x)$, where the $\mathfrak{p}$ is a function $\mathfrak{p}:[\alpha, \beta] \longrightarrow \mathbb{R}^{+}$, and then integrating the resulting identity from $\alpha$ to $v$, we see

$$
\begin{aligned}
& \frac{1}{\lambda \Gamma(\kappa)} \int_{\alpha}^{v}\left(\ln \frac{v}{x}\right)^{\kappa / \lambda-1} \mathfrak{p}(x) \mathfrak{H}(x, y) \frac{d x}{x}={ }_{H} \mathscr{J}_{1, v}^{-\kappa, \lambda}\left[\mathfrak{p} \mathfrak{S}_{1} \mathfrak{H}_{2}(v)\right] \\
& \quad-\mathfrak{H}^{\prime}(y)_{H} \mathscr{J}_{1, v}^{-\kappa, \lambda}\left[\mathfrak{p} \mathfrak{H}_{1}(v)\right]-\mathfrak{S}_{1}(y)_{H} \mathscr{I}_{1, v}^{-\kappa, \lambda}\left[\mathfrak{p} \mathfrak{H}_{2}(v)\right] \\
& \quad+\mathfrak{S}_{1}(y) \mathfrak{H}_{2}(y)_{H} \mathscr{J}_{1, v}^{-\kappa, \lambda}[\mathfrak{p}(v)] .
\end{aligned}
$$

Now multiplying (12) by $\left((\ln (v / y))^{\kappa / \lambda-1} / y \lambda \Gamma(\kappa)\right) \mathfrak{p}(y)$ for $y \in(\alpha, v)$, and then integrating the resulting identity over $(\alpha, v)$ with respect to $y$, we see

$$
\begin{aligned}
& \frac{1}{\lambda^{2} \Gamma^{2}(\kappa)} \int_{\alpha}^{v} \int_{\alpha}^{v}\left(\ln \frac{v}{x}\right)^{\kappa / \lambda-1}\left(\ln \frac{v}{y}\right)^{\kappa / \lambda-1} \mathfrak{p}(y) \mathfrak{p}(x) \mathfrak{H}(x, y) \frac{d x}{x} \frac{d y}{y} \\
& =2_{H} \mathscr{J}_{1, v}^{-\kappa, \lambda}[\mathfrak{p}(v)]_{H} \mathscr{I}_{1, v}^{-\kappa, \lambda}\left[\mathfrak{p} \mathfrak{S}_{1} \mathfrak{H}_{2}(v)\right] \\
& \quad-2_{H} \mathscr{J}_{1, v}^{-\kappa, \lambda}\left[\mathfrak{p} \mathfrak{H}_{2}(v)\right]_{H} \mathscr{I}_{1, v}^{-\kappa, \lambda}\left[\mathfrak{p} \mathfrak{S}_{1}(v)\right] .
\end{aligned}
$$

Putting $\quad \mathfrak{p}(v)=\mathfrak{g}(v) \quad$ and $\quad \mathfrak{H}_{1}(v)=\mathfrak{H}_{2}(v)=v-E(\mathscr{Z})$, $v \in(\alpha, \beta)$ in $(13)$, we see

$$
\begin{aligned}
& \frac{1}{\lambda^{2} \Gamma^{2}(\kappa)} \int_{\alpha}^{v} \int_{\alpha}^{v}\left(\ln \frac{v}{x}\right)^{\kappa / \lambda-1}\left(\ln \frac{v}{y}\right)^{\kappa / \lambda-1} \mathfrak{g}(y) \mathfrak{g}(x)(x-y)^{2} \frac{d x}{x} \frac{d y}{y} \\
& =2_{H} \mathscr{J}_{1, v}^{-\kappa, \lambda}[\mathfrak{g}(v)]_{H} \mathcal{J}_{1, v}^{-\kappa, \lambda}\left[\mathfrak{g}(v)\left(v-E(X)^{2}\right)\right] \\
& \quad-2_{H}^{\mathscr{J}_{1, v}^{-\kappa, \lambda}}[\mathfrak{g}(v)(v-E(X))]^{2} .
\end{aligned}
$$

But we can also write that as

$$
\begin{gathered}
\frac{1}{\lambda^{2} \Gamma^{2}(\kappa)} \int_{\alpha}^{v} \int_{\alpha}^{v}\left(\ln \frac{v}{x}\right)^{\kappa / \lambda-1}\left(\ln \frac{v}{y}\right)^{\kappa / \lambda-1} \mathfrak{g}(y) \mathfrak{g}(x)(x-y)^{2} \frac{d x}{x} \frac{d y}{y} \\
\leq\|\mathfrak{g}\|_{\infty}^{2} \frac{1}{\lambda^{2} \Gamma^{2}(\kappa)} \int_{\alpha}^{v} \int_{\alpha}^{v}\left(\ln \frac{v}{x}\right)^{\kappa / \lambda-1}\left(\ln \frac{v}{y}\right)^{\kappa / \lambda-1}(x-y)^{2} \frac{d x}{x} \frac{d y}{y} \\
\leq\|\mathfrak{g}\|_{\infty}^{2}\left[2 \frac{\ln (v / \alpha)^{\kappa / \lambda-1}}{\Gamma(\kappa+1)} \underset{H}{\left.\mathscr{I}_{1, v}^{-\kappa, \lambda}\left[v^{2}\right]-2\left(H_{1, v}^{\mathcal{I}^{-\kappa, \lambda}}[v]\right)^{2}\right] .}\right.
\end{gathered}
$$

To prove (ii), we can write

$$
\begin{aligned}
& \frac{1}{\lambda^{2} \Gamma^{2}(\kappa)} \int_{\alpha}^{v} \int_{\alpha}^{v}\left(\ln \frac{v}{x}\right)^{\kappa / \lambda-1}\left(\ln \frac{v}{y}\right)^{\kappa / \lambda-1} \mathfrak{g}(y) \mathfrak{g}(x)(x-y)^{2} \frac{d x}{x} \frac{d y}{y} \\
& \quad \leq \sup _{x, y \in[\alpha, v]}|x-y|^{2}\left({ }_{H} \mathcal{I}_{1, v}^{-\kappa, \lambda}[\mathfrak{g}(v)]\right)^{2} \\
& \quad=|x-y|^{2}\left({ }_{H} \mathcal{I}_{1, v}^{-\kappa, \lambda}[\mathfrak{g}(v)]\right)^{2} .
\end{aligned}
$$

Now using (14) and (16), the part (ii) of the result follows. This completes the proof.

Theorem 10. Let the continuous r.v. be $\mathscr{Z}$ with p.d.f. $\mathfrak{g}:[\alpha, \beta]$ $\longrightarrow \mathbb{R}^{+}$. Then

(i) the inequality

$$
\begin{aligned}
& { }_{H} \mathcal{J}_{1, v}^{-\kappa, \lambda}[\mathfrak{g}(v)] \sigma_{\mathscr{Z}, \omega, \lambda}^{2}+{ }_{H} \mathcal{J}_{1, v}^{-\omega, \lambda}[\mathfrak{g}(v)] \sigma_{\mathscr{Z}, \kappa, \lambda}^{2}-\left(E_{(\mathscr{X}-E(\mathscr{E}), \kappa, \lambda}(v)\right) \\
& \times\left(E_{(\mathscr{E}-E(\mathscr{Z}), \kappa, \omega}(v)\right) \leq\|\mathfrak{g}\|_{\infty}^{2}\left[\frac{(\ln (v / \alpha))^{\kappa / \lambda}}{\Gamma(\kappa+1)} \mathscr{I}_{1, v}^{-\omega, \lambda}\left[v^{2}\right]\right. \\
& \left.+\frac{\ln (v / \alpha)^{\omega / \lambda}}{\Gamma(\kappa+1)} \mathscr{I}_{H}^{-\kappa, \lambda}\left[v^{2}\right]-\left({ }_{H}^{\mathscr{J}_{1, v}^{-\kappa, \lambda}}[v]\right)\left({ }_{H}^{\mathscr{J}_{1, v}^{-\omega, \lambda}}[v]\right)\right] \text {, }
\end{aligned}
$$

holds for $\alpha<v \leq \beta, \omega \geq 0$, and $f \in L_{\infty}([\alpha, \beta])$ and for all $\kappa \geq 0$ and

(ii) the inequality

$$
\begin{aligned}
& { }_{H} \mathscr{I}_{1, v}^{-\kappa, \lambda}[\mathfrak{g}(v)] \sigma_{\mathscr{Z}, \omega, \lambda}^{2}{ }^{+}{ }_{H} \mathscr{I}_{1, v}^{-\omega, \lambda}[\mathfrak{g}(v)] \sigma_{\mathscr{E}, \kappa, \lambda}^{2}-\left(E_{(\mathscr{E}-E(\mathscr{E}), \kappa, \lambda}(v)\right) \\
& \times\left(E_{(\mathscr{X}-E(\mathscr{I}), \kappa, \omega}(v)\right) \leq(v-\alpha)^{2}\left({ }_{H}^{\mathscr{J}_{1, v}^{-\kappa, \lambda}}[\mathfrak{g}(v)]\right) \\
& \cdot\left({ }_{H}^{\mathscr{J}_{1, v}^{-\omega, \lambda}}[\mathfrak{g}(v)]\right)
\end{aligned}
$$

holds for $\alpha<v \leq \beta$ and $\omega \geq 0$.

Proof. To prove (i), we multiply $(12)$ by $\left((\ln (v / y))^{\omega / \lambda-1} /\right.$ $x \lambda \Gamma(\omega)) \mathfrak{p}(y)$ for both sides and get

$$
\begin{aligned}
& \frac{1}{\lambda^{2} \Gamma(\kappa) \Gamma(\omega)} \int_{\alpha}^{v} \int_{\alpha}^{\nu}\left(\ln \frac{\nu}{x}\right)^{\kappa / \lambda-1}\left(\ln \frac{v}{y}\right)^{\kappa / \lambda-1} \mathfrak{p}(y) \mathfrak{p}(x) \mathfrak{S}(x, y) \frac{d x}{x} \frac{d y}{y} \\
& ={ }_{H} \mathscr{J}_{1, v}^{-\kappa, \lambda}[\mathfrak{p}(v)]_{H} \mathscr{J}_{1, v}^{-\omega, \lambda}\left[\mathfrak{p}(v) \mathfrak{H}_{1}(v) \mathfrak{H}_{2}(v)\right] \\
& { }_{+}{ }_{H} \mathscr{J}_{1, v}^{-\omega, \lambda}[\mathfrak{p}(v)]_{H} \mathscr{J}_{1, v}^{-\kappa, \lambda}\left[\mathfrak{p}(v) \mathfrak{H}_{1}(v) \mathfrak{H}_{2}(v)\right] \\
& { }_{H_{H}} \mathscr{I}_{1, v}^{-\omega, \lambda}\left[\mathfrak{p}(v) \mathfrak{H}_{2}(v)\right]_{H} \mathscr{I}_{1, v}^{-\lambda, \lambda}\left[\mathfrak{p}(v) \mathfrak{H}_{1}(v)\right] \\
& { }_{-}{ }_{H} \mathcal{J}_{1, v}^{-\kappa, \lambda}\left[\mathfrak{p}(v) \mathfrak{H}_{2}(v)\right]_{H} \mathcal{I}_{1, v}^{-\omega, \lambda}\left[\mathfrak{p}(v) \mathfrak{S}_{1}(v)\right] .
\end{aligned}
$$

Consequently, part (i) follows from (14) and (15). 
Putting $\mathfrak{p}(v)=\mathfrak{g}(v), \mathfrak{H}_{1}(v)=\mathfrak{H}_{2}(v)=v-E(\mathscr{X})$, and $v \in$ $(\alpha, \beta)$ in $(19)$, we see

$$
\begin{aligned}
& \frac{1}{\lambda^{2} \Gamma(\kappa) \Gamma(\omega)} \int_{\alpha}^{v} \int_{\alpha}^{v}\left(\ln \frac{v}{x}\right)^{\kappa / \lambda-1}\left(\ln \frac{v}{y}\right)^{\omega / \lambda-1} \mathfrak{g}(y) \mathfrak{g}(x)(x-y)^{2} \frac{d x}{x} \frac{d y}{y} \\
& ={ }_{H} \mathcal{J}_{1, v}^{-\kappa, \lambda}[\mathfrak{g}(v)]_{H} \mathcal{J}_{1, v}^{-\omega, \lambda}\left[\mathfrak{g}(v)\left(v-E(\mathfrak{X})^{2}\right)\right] \\
& \quad+{ }_{H} \mathcal{J}_{1, v}^{-\omega, \lambda}[\mathfrak{g}(v)] \times{ }_{H} \mathcal{J}_{1, v}^{-\kappa, \lambda}\left[\mathfrak{g}(v)\left(v-E(\mathfrak{X})^{2}\right)\right] \\
& \quad-2_{H} \mathcal{F}_{1, v}^{-\kappa, \lambda}[\mathfrak{g}(v)(v-E(\mathfrak{X}))]_{H} \mathcal{F}_{1, v}^{-\omega, \lambda}[\mathfrak{g}(v)(v-E(\mathfrak{X}))] .
\end{aligned}
$$

But we can also write

$$
\begin{aligned}
& \frac{1}{\lambda^{2} \Gamma(\kappa) \Gamma(\omega)} \int_{\alpha}^{v} \int_{\alpha}^{v}\left(\ln \frac{v}{x}\right)^{\kappa / \lambda-1}\left(\ln \frac{v}{y}\right)^{\omega / \lambda-1} \mathfrak{g}(y) \mathfrak{g}(x)(x-y)^{2} \frac{d x}{x} \frac{d y}{y} \\
& \leq\|\mathfrak{g}\|_{\infty}^{2} \frac{1}{\lambda^{2} \Gamma^{2}(\kappa)} \int_{\alpha}^{v} \int_{\alpha}^{v}\left(\ln \frac{v}{x}\right)^{\kappa / \lambda-1}\left(\ln \frac{v}{y}\right)^{\kappa / \lambda-1}(x-y)^{2} \frac{d x}{x} \frac{d y}{y} \\
& \leq\|\mathfrak{g}\|_{\infty}^{2}\left[\frac{(\ln (v / \alpha))^{\kappa / \lambda-1}}{\Gamma(\kappa+1)}{ }_{H}^{\mathcal{F}} \mathcal{F}_{1, \nu}^{-\kappa, \lambda}\left[v^{2}\right]+\frac{(\ln (v / \alpha))^{\omega / \lambda}}{\Gamma(\omega+1)} \mathscr{I}_{H}^{-\omega, \nu}\left[v^{2}\right]\right. \\
& \left.\quad-2\left({ }_{H} \mathcal{J}_{1, v}^{-\kappa, \lambda}[v]\right)\left({ }_{H}^{\mathcal{J}_{1, v}^{-\omega, \lambda}}[v]\right)\right] .
\end{aligned}
$$

Consequently, part (i) of the result follows from (20) and (21).

To prove part (ii), we use the truth that $\sup _{v, y \in[\alpha, v]}|x-y|^{2}=$ $(x-\alpha)^{2}$ and get

$$
\begin{aligned}
& \frac{1}{\lambda^{2} \Gamma(\kappa) \Gamma(\omega)} \int_{\alpha}^{v} \int_{\alpha}^{v}\left(\ln \frac{v}{x}\right)^{\kappa / \lambda-1}\left(\ln \frac{v}{y}\right)^{\omega / \lambda-1} \mathfrak{g}(y) \mathfrak{g}(x)(x-y)^{2} \frac{d x}{x} \frac{d y}{y} \\
& \quad \leq(x-\alpha)^{2}\left({ }_{H} \mathcal{J}_{1, v}^{-\kappa, \lambda}[\mathfrak{g}(v)]\right)\left({ }_{H}^{\mathcal{F}_{1, v}^{-\omega, \lambda}}[\mathfrak{g}(v)]\right) .
\end{aligned}
$$

Consequently, part (ii) of the result follows by employing (20) and (21).

\section{Applications and Examples}

Application 11. Consider the positive functions $\mathfrak{g}$ and $\mathfrak{h}$ on $[\alpha, \beta]$ such that for every $u>\alpha, 0<_{H} \mathcal{J}_{1, v}^{-\kappa, \lambda} \mathfrak{g}^{m}{ }^{m}{ }_{H} \mathcal{J}_{1, v}^{-\kappa, \lambda} \mathfrak{h}^{m}<\infty$ with

$$
0<K \leq \frac{\mathfrak{g}(u)}{\mathfrak{h}(u)} \leq L<\infty, u \in[\alpha, \beta]
$$

where $m \geq 1$; then, for every $\kappa>0$, we have

$$
\begin{aligned}
& \left({ }_{H} \mathcal{F}_{1, v}^{-\kappa, \lambda}\left(\mathfrak{g}^{m}(u)\right)^{2 / m}+\left({ }_{H} \mathcal{F}_{1, v}^{-\kappa, \lambda}\left(\mathfrak{h}^{m}(u)\right)^{2 / m}\right.\right. \\
& \quad \geq\left[\frac{(K+1)(L+1)}{L}-2\right]\left[{ }_{H} \mathcal{F}_{1, v}^{-\kappa, \lambda}\left(\mathfrak{g}^{m}(v)\right]^{1 / m}\left({ }_{H} \mathcal{F}_{1, v}^{-\kappa, \lambda} \mathfrak{h} \mathfrak{h}^{m}(v)\right)^{1 / m} .\right.
\end{aligned}
$$

Solution 12. From (23), we see

$$
\begin{aligned}
\frac{1}{L} & \leq \frac{\mathfrak{h}(u)}{\mathfrak{g}(u)} \Rightarrow\left(\frac{1}{L}+1\right)^{m} \\
& \leq\left(\frac{\mathfrak{h}(u)}{\mathfrak{g}(u)}+1\right)^{m} \Rightarrow(L+1)^{m} \mathfrak{g}^{m}(u) \\
& \leq L^{m}(\mathfrak{h}+\mathfrak{g})^{m}(u) .
\end{aligned}
$$

In a similar way, we see

$$
K \leq \frac{\mathfrak{g}(u)}{\mathfrak{h}(u)} \Rightarrow(K+1)^{m} \mathfrak{g}^{m}(u) \leq(\mathfrak{h}+\mathfrak{g})^{m}(u) .
$$

Consequently, multiplying these equations by $(\ln (v / u))^{\kappa / \lambda-1} / u \lambda \Gamma(\kappa)$ for $u \in(\alpha, v)$ and then integrating the resulting identity over $(\alpha, v)$ with respect to $u$ yield

$$
\begin{gathered}
\left({ }_{H} \mathcal{F}_{1, v}^{-\kappa, \lambda}\left(\mathfrak{g}^{m}(u)\right)\right)^{1 / m} \leq \frac{L}{L+1}\left((\mathfrak{g}+\mathfrak{h})^{m}(u)\right)^{1 / m}, \\
\left({ }_{H} \mathcal{I}_{1, v}^{-\kappa, \lambda}\left(\left(\mathfrak{h}^{m}(u)\right)\right)^{\frac{1}{m}} \leq \frac{1}{K+1}\left((\mathfrak{g}+\mathfrak{h})^{m}(u)\right)\right)^{\frac{1}{m}} .
\end{gathered}
$$

Now on multiplying (27) and (28), we see

$$
\begin{aligned}
& \frac{(K+1)(L+1)}{L}\left({ }_{H} \mathcal{F}_{1, v}^{-\kappa, \lambda}\left(\left(\mathfrak{g}^{m}(u)\right)\right)^{1 / m}\left({ }_{H} \mathcal{J}_{1, v}^{-\kappa, \lambda}\left(\mathfrak{h}^{m}(u)\right)\right)^{1 / m}\right. \\
& \quad \leq\left({ }_{H} \mathcal{F}_{1, \nu}^{-\kappa, \lambda}\left((\mathfrak{g}+\mathfrak{h})^{m}(u)\right)\right)^{2 / m} .
\end{aligned}
$$

Consequently, the result follows by using Minkowski's integral inequality on the right-hand side of (29).

Example 1. Consider the function $\mathfrak{g}(u)=(\ln (u / \alpha))^{\eta / \lambda-1}$, we see

$$
{ }_{H} \mathcal{J}_{\alpha, u}^{-\kappa, \lambda}\left(\ln \frac{u}{\alpha}\right)^{\eta / \lambda-1}=\frac{1}{\lambda \Gamma(\kappa)} \int_{\alpha}^{v}\left(\ln \frac{u}{w}\right)^{\kappa / \lambda-1}\left(\ln \frac{w}{\alpha}\right)^{\eta / \lambda-1} \frac{d w}{w} .
$$

Choosing $\tau=\ln (u / w) / \ln (u / \alpha)$, for $\tau \in(\alpha, \beta]$ with $\eta$, $\kappa>0$, we see with the help of (2) that

$$
\begin{aligned}
{ }_{H} \mathcal{F}_{\alpha, u}^{-\kappa, \lambda}\left(\ln \frac{u}{\alpha}\right)^{\kappa / \lambda-1} & =\left(\ln \frac{u}{\alpha}\right)^{(\kappa+\eta) / \lambda-1} \frac{1}{\lambda \Gamma(\kappa)} \int_{0}^{1}(1-\tau)^{\eta / \lambda-1}(1-\tau)^{\kappa / \lambda-1} d \tau \\
& =\left(\ln \frac{v}{w}\right)^{\kappa / \lambda-1} \frac{\beta(\kappa / \lambda, \eta / \lambda)}{\lambda \Gamma(\kappa)} .
\end{aligned}
$$

\section{Data Availability}

No data were used in this study.

\section{Conflicts of Interest}

The authors declare that there is no conflict of interest. 


\section{References}

[1] S. Erden, H. Budak, M. Z. Sarikaya, S. Iftikhar, and P. Kumam, "Fractional Ostrowski type inequalities for bounded functions," Journal of inequalities and applications, vol. 2020, no. 1, Article ID 123, 2020.

[2] A. H. Ganie, "Some new approach of spaces of non-integral order," Journal of Nonlinear Sciences and Applications, vol. 14, no. 2, pp. 89-96, 2021.

[3] A. H. Ganie, "Lacunary sequences with almost and statistical convergence," Annals of Communications in Mathematics, vol. 3, no. 1, pp. 46-53, 2020.

[4] A. H. Ganie and D. Fathima, "Almost convergence property of generalized Riesz spaces," Journal of Applied Mathematics and Computation, vol. 4, no. 4, article 249253, 2020.

[5] A. H. Ganie and M. A. Syed, "On some new generalized difference sequence space of fuzzy numbers and statistical convergence," Journal of Applied Mathematics and Computation, vol. 4, pp. 4-6, 2015.

[6] P. A. Naik, M. Yavuz, S. Qureshi, J. Zu, and S. Townley, "Modeling and analysis of COVID-19 epidemics with treatment in fractional derivatives using real data from Pakistan," European Physical Journal Plus, vol. 135, no. 10, p. 795, 2020.

[7] F. Qi, "Several integral inequalities," Journal of Inequalities in Pure and Applied Mathematics, vol. 1, no. 2, pp. 1-9, 2000.

[8] N. A. Sheikh and A. H. Ganie, "On the space of $\lambda$-convergent sequence and almost convergence," Thai Journal of Mathematics, vol. 2, no. 11, pp. 393-398, 2013.

[9] A. Akkurt, Z. Kirtay, and H. Yıldırım, "Generalized fractional integrals inequalities for continuous random variables," Journal of Probablity Statistics, vol. 2015, article 958980, pp. 1-7, 2015.

[10] G. A. Anastassiou, M. R. Hooshmandasl, A. Ghasemi, and F. Moftakharzadeh, "Montgomery identities for fractional integrals and related fractional inequalities," Journal of Inequalities in Pure and Applied Mathematics, vol. 10, no. 4, pp. 1-6, 2009.

[11] S. G. Samko, A. A. Kilbas, and O. I. Marichev, Fractional Integrals and Derivatives, Theory and Applications, Gordon and Breach, Yverdon, Switzerland, 1993.

[12] H. Yıldırım and Z. Kırtay, "Ostrowski inequality for generalized fractional integral and related inequalities," Malaya Journal of Matematik, vol. 2, no. 3, pp. 322-329, 2014.

[13] Z. Dahmani, "New applications of fractional calculus on probabilistic random variables," Acta Mathematica Universitatis Comenianae, vol. 86, no. 2, pp. 299-307, 2017.

[14] Z. Dahmani, "New identities and lower bounds for random variables: applications for CUD and beta distributions," ROMAI Journal, vol. 15, no. 1, pp. 25-35, 2019.

[15] Z. Dahmani, A. Khameli, M. Bezziou, and M. Z. Sarikaya, "Some estimations on continuous random variables involving fractional calculus," International Journal of Analysis and Applications, vol. 15, no. 1, pp. 8-17, 2017.

[16] Z. Dahmani, "Fractional integral inequalities for continuous r.v.s," Malaya Journal of Matematik, vol. 2, no. 2, pp. 172179, 2014.

[17] M. Houas, "Some estimations on continuous random variables for $(\mathrm{k}, \mathrm{s})$-fractional integral operators," Moroccan Journal of Pure and Applied Analysis, vol. 6, no. 1, pp. 143-154, 2020.

[18] P. A. Naik, K. M. Owolabi, M. Yavuz, and J. Zu, "Chaotic dynamics of a fractional order HIV-1 model involving AIDS- related cancer cells," Chaos, Solitons \& Fractals, vol. 140, article 110272, 2020.

[19] G. H. Abdul, "New approach for structural behavior of variables," Journal of Nonlinear Sciences \& Applications (JNSA), vol. 14, no. 5, pp. 351-358, 2021.

[20] A. A. Kilbas, H. M. Srivastava, and J. J. Trujillo, "Theory and applications of fractional differential equations," in North-Holland Mathematics Studies, vol. 204, Elsevier, Amsterdam, 2006.

[21] P. Kumar, "Moment inequalities of a r.v. defined over a finite interval," Journal of Inequalities in Pure and Applied Mathematics, vol. 3, no. 3, pp. 1-24, 2002.

[22] R. Mustapha and S. E. Soubhy, "On some generalizations of the beta function in several variables," Turkish Journal of Mathematics, vol. 45, pp. 820-842, 2021.

[23] P. A. Naik, J. Zu, and K. Owolabi, "Modeling the mechanics of viral kinetics under immune control during primary infection of HIV-1 with treatment in fractional order," Physica A: Statistical Mechanics and its Applications, vol. 545, article 123816, 2020.

[24] P. A. Naik, J. Zu, and K. Owolabi, "Global dynamics of a fractional order model for the transmission of HIV epidemic with optimal control," Chaos, Solitons \& Fractals, vol. 138, article 109826, 2020.

[25] M. Niezgoda, "New bounds for moments of continuous random variables," Computers and Mathematics with Applications, vol. 60, no. 12, pp. 3130-3138, 2010.

[26] M. I. Troparevsky, S. A. Seminara, and M. A. Fabio, "A review on fractional differential equations and a numerical method to solve some boundary value problems," Mathematics, vol. 2019, 2020.

[27] V. L. Chinchane and D. B. Pachpatte, "On some Grüss-type fractional inequalities using Saigo fractional integral operator," Journal of Fractional Calculus and Applications, vol. 2014, no. 12, pp. 1-9, 2014.

[28] V. L. Chinchane and D. B. Pachpatte, "On some integral inequalities using Hadamard fractional integral," Malaya journal of matematik, vol. 1, no. 1, pp. 62-66, 2012.

[29] S. B. Chen, S. Rashid, M. A. Noor, R. Ashraf, and Y. M. Chu, "A new approach on fractional calculus and probability density function," AIMS Mathematics, vol. 6, no. 5, pp. 7041-7054, 2020.

[30] J. Hadamard, "Essai sur l'etude des fonctions donn'e'es par leur d'eveloppement de Taylor," Journal of pure and applied mathematics, vol. 8, pp. 101-186, 1892.

[31] O. M. Khellaf and V. L. Chinchane, "Continuous random variables with Hadamard fractional integral," Tamkang Journal of Mathematics, vol. 50, no. 2, pp. 103-109, 2019.

[32] M. Houas and O. M. Khellaf, "Existence and uniqueness results for a coupled system of Hadamard fractional differential equations with multi-point boundary conditions," Facta Universitatis, Series: Mathematics and Informatics, vol. 35, no. 3, pp. 843-856, 2020.

[33] P. Agarwal, S. Sever, D. M. Jleli, and B. Samet, Advances in Mathematical Inequalities and Applications, Birkhaüuser, Basel, 2018.

[34] P. Agarwal, J. Tariboon, and S. K. Ntouyas, "Some generalized Riemann-Liouville k-fractional integral inequalities," Journal of Inequalities and Applications, vol. 2016, no. 1, 13 pages, 2016.

[35] A. Alsaedi, B. Ahmad, and M. Alghanmi, "Extremal solutions for generalized Caputo fractional differential equations with Steiltjes-type fractional integro-initial conditions," Applied Mathematics Letters, vol. 91, pp. 113-120, 2019. 
[36] R. Zafar, M. u. Rehman, and M. Shams, "On Caputo modification of Hadamard-type fractional derivative and fractional Taylor series," Advances in Difference Equations, vol. 2020, no. $1,2020$.

[37] H. H. G. Hashem and H. O. Alrashidi, "Qualitative analysis of nonlinear implicit neutral differential equation of fractional order," AIMS Mathematics, vol. 6, no. 4, pp. 3703-3719, 2021.

[38] G. Rahman, K. S. Nisar, and T. Abdeljawad, "Certain Hadamard proportional fractional integral inequalities," Mathematics, vol. 8, no. 4, p. 504, 2020. 